\title{
DNA-mediated electron transfer: Chemistry at a distance
}

\author{
Jacqueline K. Barton \\ Arnold and Mabel Beckman Laboratories of Chemical Synthesis, California \\ Institute of Technology, Pasadena, CA 91125, USA
}

\begin{abstract}
The DNA double helix, containing a $\pi$-stacked array of base pairs in its core, represents a unique and efficient medium for long-range charge transport. DNA assemblies have been constructed containing tethered metallointercalators, and these provide chemically well-defined systems through which to probe the DNA $\pi$-stack. Using both spectroscopy and chemical assays of reactivity, we find electron transfer reactions mediated by the DNA base pairs to occur over long molecular distances. The structure of DNA facilitates chemistry at a distance. Importantly, these long-range reactions depend sensitively upon base pair stacking, and hence are modulated by and report on the characteristic stacking within the double helix.
\end{abstract}

\section{INTRODUCTION}

The DNA double helix is a remarkable polymeric structure which serves as our biological library, encoding all the information of the cell.(1) But the DNA double helix, as a molecular $\pi$-stacked array, is also chemically a unique structure. While the periphery of this double helical structure contains the negatively charged sugar phosphate backbone, in the center of the DNA double helix, the aromatic heterocyclic base pairs are stacked one with another. This base pair stacking contributes substantial stability to the DNA duplex. We are interested in understanding some of the chemical characteristics and consequences of this unique $\pi$-stacked biopolymeric structure. Analogous doped $\pi$-stacked arrays in the solid state are known to be efficient conductors along the stacking direction. Our interest is in exploring whether or not such a molecular $\pi$-stacked array might serve as a particularly efficient medium to promote charge transport over long molecular distances.

The question of whether or not radicals, electrons and (electron) holes, migrate across the DNA helix is one that has been asked for over thirty years.(2) This question is an important one to consider in a biological context: whether radicals migrate through a DNA helix over two base pairs or two hundred is debated extensively by radiation biologists.(3) Indeed, such charge mobility has enormous implications in the context of understanding mechanisms of mutagenesis and carcinogenesis. Theorists have similarly debated mechanisms of hole hopping, tunneling, superexchange or band delocalization.(4) Recent chemical experiments have focused on the utilization of DNA assemblies with donors and acceptors bound non-covalently $(5,6)$ or covalently $(7-9)$ to the DNA helix in which electron transfer may be probed spectroscopically.

Here we describe studies from our laboratory where the focus is on donors and acceptors bound within DNA assemblies at fixed positions in covalent complexes. Such studies enable us to describe systematically how DNA-mediated electron transfer depends upon the intervening distance between donors and acceptors, the intervening sequence, and the manner in which the donor and acceptor are coupled into the DNA $\pi$-stack. We utilize both spectroscopic methods and chemical reactivity to examine long-range charge transport and its consequences. Importantly our studies all focus on the application of metallointercalators. These complexes, in binding tightly within the $\pi$-stack of DNA, interacting directly with the DNA base pairs, provide a specific probe of $\pi$-stacking itself. Our studies in fact underscore the sensitivity of DNA-mediated charge transport chemistry to $\pi$-stacking; both the stacking of donor and acceptor, as well as the stacking characteristics of the DNA double helix itself.

\section{DNA as a bridge between a bound ruthenium and rhodium intercalator.}

A range of octahedral metallointercalators have been designed in our laboratory and their binding characteristics to DNA have been well characterized.(10) These metal complexes bind DNA with 
affinities of $10^{6}$ to $10^{8} \mathrm{M}^{-1}$. Ruthenium intercalators containing the dipyridophenazine (dppz) ligand have been particularly useful as luminescent probes of DNA.(11) Phenanthrenequinone diimine (phi) complexes of rhodium have been valuable in exploring site-specific recognition by small molecules.(12) Once bound to DNA by intercalation, with photoactivation, these phi complexes promote DNA direct strand cleavage and, in so doing, mark their sites of binding. The sites targeted by the phi complexes can be tuned by varying the ancillary ligands. As an example, $\left(\mathrm{Me}_{2}\right.$ trien) Rhphi ${ }^{3+}$ has been shown to specifically target 5'-TGCA-3' through an ensemble of non-covalent contacts between $\mathrm{Me}_{2}$ trien and the DNA major groove.(13) $\mathrm{Rh}(\mathrm{phi})_{2} \mathrm{bpy}^{3+}$ is, in contrast, relatively sequence-neutral in its binding to DNA.(14)

These ruthenium and thodium complexes may be used in concert to explore DNA-mediated electron transfer by spectroscopic techniques. In studies with non-covalently bound $\mathrm{Ru}(\mathrm{phen})_{2} \mathrm{dppz}^{2+}$ and $\mathrm{Rh}(\mathrm{phi})_{2} \mathrm{bpy}^{3+}$, efficient quenching of ruthenium luminescence is found at low loadings of the sequenceneutral rhodium intercalator, $\mathrm{Rh}(\mathrm{phi})_{2} \mathrm{bpy}^{3+} .(5,15)$ Transient absorption studies established the quenching to be the result of photoinduced electron transfer. $(16,17)$ Moreover, this quenching appeared to be quite rapid $\left(>3 \times 10^{10} \mathrm{~s}^{-1}\right)$. As a result, several laboratories proposed that this quenching arises as a result of the cooperative clustering of intercalators on the helix.(18) Recently circular dichroism and NMR studies have, however, indicated that the intercalators bind non-cooperatively to the DNA duplex.(19) Given a lack of cooperativity in binding, an alternate interpretation for the fast quenching would involve electron transfer over long range between the metallointercalators. DNA-binding molecules which associate in a groove-bound fashion show no similar fast photoinduced quenching behavior.(5)

That this photoinduced electron transfer occurs over long range is best demonstrated in a DNA assembly where the intercalators are tethered covalently to opposing ends of the duplex.(7) Figure 1 illustrates such an assembly. The ruthenated duplex oligomer, made by hybridization of the ruthenium-modified 15 -mer strand to an unmodified complement, showed significant luminescence, comparable to that of ruthenium non-covalently bound. In contrast, in the mixed-metal 15-mer oligonucleotide duplex, containing the ruthenium intercalator tethered to the $5^{\prime}$-end of one strand and the rhodium intercalator tethered to the 5'-end of the other strand, no luminescence was evident. Photocleavage studies on the rhodium-modified duplex indicated intercalation of the intercalators at most two base pairs in from the end. Hence, in the mixed-metal assembly, where the donor and acceptor intercalators are separated by $>$ $41 \AA$ along the helix axis, fast photoinduced electron transfer appears to occur over long range.

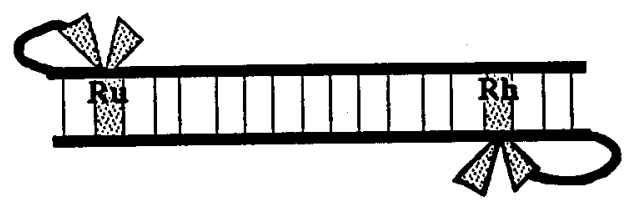

Fig. 1. A mixed-metal DNA assembly with tethered intercalators.

It is noteworthy that these results were fully consistent with studies using non-covalent intercalators. Control studies using non-intercalated, but tethered ruthenium and rhodium complexes showed no quenching. These results gave us the first indication of the requirement of intercalative stacking for efficient charge transport. However, in the modified assembly containing the metallointercalators, the exciting result is in, in fact, an observation of the absence of luminescence. Is this apparent efficient quenching particular to the ruthenium system, a complex which undergoes luminescence quenching in water? Can long-range electron transfer between DNA intercalators be probed more directly and systematically?

\section{DNA as a bridge between ethidium and rhodium intercalators}

To probe whether DNA-mediated electron transfer between bound intercalators could be generalized, experiments were carried out using tethered ethidium as the photoinduced donor. Ethidium represents a well-characterized classical organic intercalator. Figure 2 illustrates an assembly prepared in our laboratory.(20) 


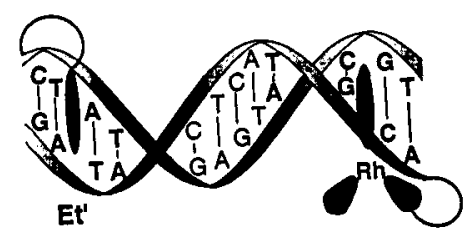

Fig. 2. A DNA assembly with tethered ethidium and rhodium intercalators.

Ethidium shares some characteristics with the ruthenium intercalator. The reduction potential for photoexcited ethidium is quite close to that of photoexcited Ru(II). Both intercalators bind to the DNA duplex with high affinity (the binding constant for the ruthenium analog is expected to be approximately an order of magnitude higher than for ethidium). As with the ruthenium intercalator, quenching of ethidium fluorescence by non-covalently bound rhodium occurs on a fast time scale $\left(>3 \times 10^{9} \mathrm{~s}^{-1}\right)$. Here, however, the efficiency of quenching is somewhat less than that for quenching of ruthenium emission.

Significant quenching of tethered ethidium emission is evident in the assemblies containing tethered rhodium. In a 10 -mer duplex, where the donor/acceptor separation is approximately $20 \AA$, approximately $30 \%$ quenching is observed, whereas for a 13-mer with a separation of about $30 \AA$, 10 $15 \%$ quenching is seen. In all cases, the quenching appears to occur on a time scale which is fast compared to the resolution of the instrumentation $\left(>10^{9} \mathrm{~s}^{-1}\right)$. Thus, as in the mixed-metal intercalator system, here too, we observe fast photoinduced electron transfer at long range between donors and acceptors intercalated in a DNA duplex.

Also here, the photoinduced electron transfer depends sensitively upon stacking, not just of stacking of the donor and acceptor, but also upon the intervening base pair. This sensitivity to stacking is perhaps best illustrated in a mismatch experiment. While $24 \%$ quenching is observed in the 11-mer duplex containing tethered ethidium and rhodium, if a TA base pair is mutated to a CA base pair at the center of the duplex, this photoinduced quenching is essentially lost (the fraction quenched is $4 \%$ ). In contrast, if the TA base pair is mutated to a GA, which is well stacked in a DNA helix based upon NMR studies, $28 \%$ quenching is observed. This mismatch experiment also serves to establish that the quenching must be mediated by the DNA helix and depends upon the characteristic sequence which intervenes between donor and acceptor; the quenching cannot arise as a result of an interaction of donor and acceptor outside of the duplex due to fraying.

Therefore, these studies, again, are consistent with a DNA-mediated electron transfer reaction which can arise over long range. The reaction is apparently only weakly sensitive to distance yet is exquisitely sensitive to stacking.

\section{Photoinduced damage to DNA from a distance with rhodium intercalators as oxidants}

The DNA double helix not only can serve as a bridge but also as a reactant in long-range electron transfer chemistry. In this context, one may consider more directly issues of importance with respect to delineating aspects of radical damage to DNA. If long-range electron transfer through DNA occurs, then one ought to be able to carry out such DNA-mediated electron transfer chemistry to promote oxidative damage to a DNA base from a distance.

Figure 3 illustrates a DNA assembly constructed to explore oxidative damage to DNA from a remote position.(21) In this assembly, a rhodium intercalator has been tethered to the 5 '-end of one strand with a tether length sufficient to allow intramolecular intercalation. That this intercalation is indeed intramolecular is determined in photocleavage experiments. But the phi complexes of rhodium can also serve as potent photooxidants when irradiated at lower energy $(\geq 360 \mathrm{~nm})$. Hence, we may apply the tethered rhodium complex to oxidize the DNA duplex directly. Based upon empirical studies with a range of photooxidants, as well as theoretical calculations, the site most easily oxidized on DNA is the guanine base, in particular the 5'-G of guanine doublets or triplets.(22) In the assembly illustrated, then, we examined whether the tethered rhodium complex could be utilized to promote oxidative damage to 
5'-GG-3' doublets $17 \AA ̊$ or $34 \AA$ away from the site of intercalation.(21)

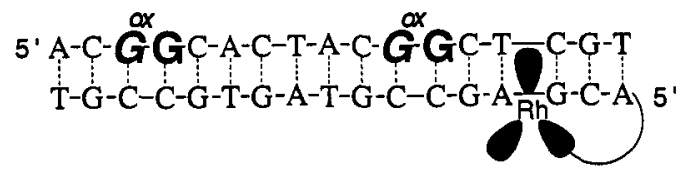

Fig. 3. A DNA assembly to oxidize 5'-GG'-3' doublets from a distance.

What we observed in this and other assemblies is that the rhodium intercalator could promote oxidative damage to DNA from a distance. The efficiency of such oxidative damage was, in fact, comparable for the rhodium complex tethered to the end of the helix or non-covalently bound at a distribution of distances. Moreover, the yield of such oxidative damage appeared to be relatively insensitive to the distance separating the 5'-GG-3' doublet from the rhodium intercalator. Our most recent experiments have indicated that the photoinjected hole, once introduced into the helix by the rhodium intercalator, can promote oxidative damage to DNA over $100 \AA$ away.(23)

Enzymatic digestion of the damaged DNA followed by HPLC analysis shows that the base product of this oxidative damage is 8-oxo-guanine. Interestingly, 8-oxo-guanine is a common oxidative lesion to cellular DNA. We consider that upon oxidation by one electron, the guanine radical is formed. Thereafter subsequent chemistry, trapping the radical by dioxygen and/or water must then occur to give the final stable product. The mechanistic details of how this irreversible oxidative damage to DNA arises need still to be established.

While the yield of this damage appears to be insensitive to distance, the yield of reaction is modulated by the intervening DNA hase pair stack. This sensitivity to base stacking is best illustrated in studies of oxidative damage in assemblies containing intervening DNA bulges.(24) We examined specifically the ratio of oxidative damage at a distal to proximal 5'-GG-3' doublet, in the presence and absence of an intervening -ATA- bulge. Such a bulge has been structurally well-characterized and shown to promote a local disruption of the stacked base pairs of DNA. We observe that in the assembly containing the bulged segment, the ratio of distal to proximal 5'-GG-3' damage is diminished 6-fold compared to the ratio observed with a well-stacked B DNA duplex. It is noteworthy also that in the bulged DNA assembly, if anything, the rhodium to guanine distance is shortened owing to kinking of the DNA duplex around the bulge; despite the shorter distance, however, the oxidative damage from afar is less efficient.

Overall, then, just as we observed in spectroscopic studies with DNA as a bridge, here, too, with DNA as a reactant, electron transfer chemistry with a metallointercalator can occur over long molecular distances. From these studies, we are not able to determine, however, the rate of electron transfer as a function of distance. We can establish only that this rate must be faster than the trapping time of the initial one-electron oxidized product, that is, the guanine radical. Importantly, the yield of this longrange electron transfer chemistry appears to be relatively insensitive to distance, yet exquisitely sensitive to the base pair stack.

\section{Long-range oxidative damage to DNA in assemblies containing tethered Ru(III)}

The rhodium oxidation chemistry has been valuable in demonstrating for the first time in a chemically well-defined system that oxidative damage to DNA could be promoted from a distance. But the quantum yields of this rhodium chemistry, indeed the efficiencies for all of the rhodium photochemistry, are quite low. We were interested in developing more efficient long-range reactions. Furthermore, we were interested in exploring how general this chemistry might be and whether other intercalators, in particular ground state oxidants, could be utilized in carrying out oxidative damage to DNA over long range.

To test the reactivity of a ground state, intercalating oxidant, we applied a "flash quench methodology" developed first in studies of protein electron transfer.(25) In this strategy, we utilized our avid ruthenium intercalators, $\mathrm{dpp} z$ complexes of $\mathrm{Ru}(\mathrm{II})$, to generate $\mathrm{Ru}(\mathrm{III})$, a potent oxidant, by oxidative quenching. Photoexcited $\mathrm{Ru}(\mathrm{II})$ bound to $\mathrm{DNA}$ can be quenched by electron transfer to the groove 
bound species, $\mathrm{Q}=\mathrm{Ru}\left(\mathrm{NH}_{3}\right)_{6}{ }^{3+}$, methyl viologen, and $\mathrm{Co}\left(\mathrm{NH}_{3}\right)_{5} \mathrm{Cl}^{2+}$. This quenching process generates $\mathrm{Ru}(\mathrm{III})$ in situ and this ground state oxidant, intercalated into the helix, can promote oxidative damage to DNA.

Utilizing this strategy, we were able to apply transient absorption spectroscopy to identify, for the first time, the neutral guanine radical in duplex DNA as the primary oxidative intermediate.(26) The transient spectrum, characteristic of this radical, was generated by photoexcitation of Ru(phen) $)_{2} \mathrm{dppz}^{2+}$ bound to poly $\mathrm{dGdC}$ and quenched by the spectroscopically silent $\mathrm{Ru}\left(\mathrm{NH}_{3}\right)_{6}{ }^{3+}$. The final irreversible product of this chemistry is also 8-oxo-G. Noteworthy, however, is that the yield of this damage can be more than 10,000-fold greater than that seen with rhodium photochemistry depending upon the quencher employed.

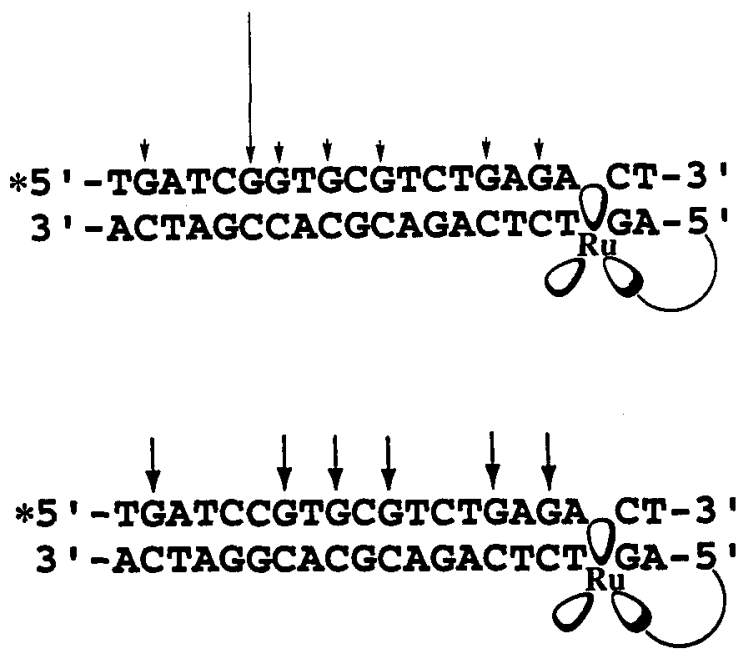

Fig. 4. Two ruthenated DNA assemblies, one with (top) and one without (bottom) a 5'-GG-3' doublet. Sites and relative efficiences of oxidation are given by the arrows.

The intercalating $\mathrm{Ru}$ (III) oxidant, furthermore, like phi complexes of rhodium, can promote oxidative damage to DNA from a distance.(27) Figure 4 illustrates two assemblies in which oxidative damage to DNA was explored. Here, a dimethyl-dipyridophenazine complex of ruthenium was appended to the end of the two DNA duplexes. In the first, a single 5'-GG-3' doublet was included in the sequence; in this case oxidative damage was evident at the $5^{\prime}-\mathrm{G}$ of the 5'-GG-3' doublet. But when a single base mutation was made in this DNA assembly, such that only single $G$ sites were present, oxidative damage was observed essentially equally at all guanine positions. Hence, the yield of oxidative damage does not appear to depend sensitively upon distance, nor upon reactive characteristics which are particular to a 5'GG-3' site. Instead, the site oxidized depends upon base oxidation potential. Thus, the difference in pattern of oxidation across these two sequences shows that the intensity of oxidation to first order depends not upon how the hole is trapped at different sites on the DNA duplex, but instead upon the ease of oxidation of a given sequence. The result graphically underscores the notion of migration of the injected hole across the DNA duplex on a time scale which is fast compared with trapping of the hole to form the irreversible oxidized product.

Oxidative repair of thymine dimers in DNA duplexes by long-range electron transfer with tethered rhodium intercalators

We were interested in applying long-range electron transfer to carry out another reaction on DNA which was more simply and directly triggered by electron transfer than was the guanine oxidation chemistry. In this context, the oxidative repair of thymine dimers represented an excellent system for study. Moreover, since the thymine dimer represents the primary photochemical lesion of the cell, its repair is of therapeutic importance. 
In bacterial systems, the enzyme photolyase, containing a bound flavin cofactor, repairs thymine dimers in a reductive catalytic cycle upon photoactivation with visible light.(28) Model studies on thymine dinucleotides had shown that thymine dimers could also be reversed in an oxidative cycle, given the instability of the cationic cyclobutane radical intermediate.(29) We, therefore, considered that phi complexes of rhodium, given their high oxidative potential and their photoactivation with visible light, (30) might be particularly amenable to studies of oxidative thymine dimer repair. Moreover, by incorporating tethered rhodium intercalators into assemblies containing thymine dimers, we could explore a reaction on DNA from a distance triggered cleanly by electron transfer.(31)

We first examined the repair of thymine dimers in DNA duplexes by non-covalently bound rhodium intercalators. We observed in HPLC experiments that, upon photoactivation with visible light, the rhodium complex could promote quantitative repair of the thymine dimers to the native unmodified form. The reaction, furthermore, yielded no secondary products and could be carried out with sunlight. Indeed, consistent with the triggering mechanism, quantitative repair could be accomplished using catalytic concentrations of rhodium (one rhodium per ten DNA duplexes). As with the guanine oxidation chemistry, however, the overall photoefficiency of the rhodium oxidation is quite low.

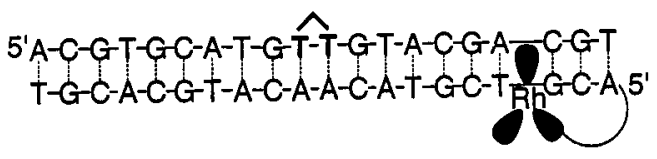

Fig. 5. The oxidative repair of a thymine dimer at a distance by a tethered rhodium intercalator.

Figure 5 shows an assembly in which we then tested the oxidative repair of a thymine dimer in DNA from a distance. We constructed DNA duplexes containing a single thymine dimer site-specifically incorporated in the center of the DNA oligomer. In these DNA duplexes, the rhodium intercalator was covalently tethered to the opposite strand. In this system, too, we were able to observe the quantitative repair of the thymine dimer. As with our other systems of study, we find the efficiency of thymine dimer repair to be relatively sensitive to the distance separating the thymine dimer from the intercalated rhodium. Quantitative repair can proceed over $>30 \AA$. Interestingly, and in contrast to long-range guanine oxidation, more than an order of magnitude decrease in repair efficiency is obtained with covalently bound rhodium compared with the non-covalent intercalator. We expect that this difference depends upon the stacking of the thymine dimer within the duplex, as well, perhaps, as with stacking of the covalently bound intercalator. It is clear, however, that stacking is a very sensitive parameter in this system as well. When experiments are carried out with a bulged base intervening between the rhodium intercalator and the thymine dimer, substantial decreases in repair efficiency are observed.

\section{CONCLUSIONS}

Studies described here represent experiments combining synthetic transition metal chemistry and biophysical chemistry designed to explore long-range electron transfer chemistry mediated by the DNA double helix. By systematically appending donors and/or acceptors to DNA duplexes and measuring electron transfer reactions, utilizing spectroscopic methods and chemical assays of reactivity, we can begin to delineate those factors which are important in establishing DNA-mediated electron transfer. From our studies, we have learned again and again that stacking is a key parameter modulating longrange chemistry, whether it is the stacking of the donor, the stacking of the acceptor, or the intervening stack of the DNA duplex. Chemistry at a distance may indeed be accomplished, but it depends upon the structure of the intervening $\pi$-stack. It is clear that the issue of electron mobility through DNA is one that bears careful consideration in developing an understanding of cellular DNA damage and its control.

Importantly, our studies have focused on exploiting intercalating moieties as probes. Metallointercalators provide an important, perhaps unique, probe of the DNA $\pi$-stack in this context. We are excited about the applications of this probe of the DNA base stack in developing new DNAbased diagnostics, and in fundamental examinations of DNA dynamics as a function of sequence and protein interaction. 
But there is much that we still do not understand. Experiments to determine rates of these fast longrange electron transfer reactions will be essential to carry out and efforts in that direction are currently ongoing. Also important to establish are quantitative parameters through which to gauge stacking, whether it be stacking as a function of sequence or the stacking of individual donors and acceptors. We also need to develop methodologies to determine the energetics for these differing reactions, redox potentials of individual bases, donors, acceptors and within the context of the DNA double helix. Once these different experimental values are better understood and described, we can begin to use these parameters to develop and test a theoretical framework for this electron transfer chemistry. Lastly, it is important to determine whether these and other electron transfer reactions mediated by the double helix are important physiologically. Does nature protect, or perhaps even exploit, DNA-mediated electron transfer reactions within the cell?

\section{ACKNOWLEDGMENTS}

I am grateful to the NIH for their financial support of this work (GM33309 and GM49216). I am also indebted to my coworkers and collaborators for their efforts in the exploration of this chemistry.

\section{REFERENCES}

1. W. Saenger. Principles of Nucleic Acid Structure, (Ed.: C. R. Cantor), Springer-Verlag, New York (1984).

2. a) R. S. Snart. Trans. Faraday Soc. 59, 754-760 (1963); b) D. D. Eley and D. I. Spivey. Trans. Faraday Soc. 58, 411.415 (1962).

3. P. O'Neill and E. M. Fielden. Adv. Radiat. Biol. 17, 53-120 (1993).

4. a) D. Dee and M. E. Baur. J. Chem. Phys. 60, 541-560 (1974), b) A. K. Felts, W. T. Pollard and R. A. Friesner. J. Phys. Chem. 99, 2929-2940 (1995). c) S. Priyadarshy, S. M. Risser and D. N. Beratan. J. Phys. Chem. 100, 17678-17682 (1996). c) S. M. Risser, D. N. Beratan and T. J. Meade. J. Am. Chem. Soc. 115, 2508-2510 (1993). d) D. N. Beratan, S. Priyadarshy and S. M. Risser. Chemistry \& Biology 4, 3-8 (1997).

5. a) J. K. Barton, C. V. Kumar and N. J. Turro. J. Am. Chem. Soc 108, 6391-6393 (1986). b) M. D. Purugganan, C. V. Kumar, N. J. Turro and J. K. Barton. Science 241, 1645-1649 (1988). b) C. J. Murphy, M. R. Arkin, N. D. Ghatlia, S. H. Bossmann, N. J. Turro and J. K. Barton. Proc. Natl. Acad. Sci. USA 91, 5315-5319 (1994).

6. a) P. Fromherz and B. Rieger. J. Am. Chem. Soc. 108, 5361-5362 (1986). b) A. M. Brun and A. Harriman. ibid. 116, 10383-10393 (1994).

7. C. J. Murphy, M. R. Arkin, Y. Jenkins, N. D. Ghatlia, S. H. Bossmann, N. J. Turro and J. K. Barton. Science 262, 1025-1029 (1993).

8. a) T. J. Meade and J. F. Kayyem. Angew. Chem. 107, 358-360 (1995). b) T. J. Meade and J. F. Kayyem. Angew. Chem. Int. Ed. Engl. 34, 352-354 (1995).

9. F. D. Lewis, T. Wu, Y. Zhang, R. L. Letsinger, S. R. Greenfield and M. R. Wasielewski. Science 277,673-676 (1997).

10. T. W. Johann and J. K. Barton. Phil. Trans. R. Soc. Lond. A 354, 299-324 (1996).

11. A. E. Friedman, J.-C. Chambron, J.-P. Sauvage, N. J. Turro and J. K. Barton. J. Am. Chem. Soc. 112, $4960-4962$ (1990).

12.

a) A. Sitlani, C. M. Dupureur and J. K. Barton. J. Am. Chem. Soc. 115, 12589-12590 (1993). b) R. H. Terbrueggen and J. K. Barton. ibid. 34, 8227-8234 (1995).

13. B. P. Hudson, C. M. Dupureur and J. K. Barton. J. Am. Chem. Soc. 117, 9379-9380 (1995).

14. A. Sitlani, E. C. Long, A. M. Pyle and J. K. Barton. J. Am. Chem. Soc. 114, 2303-2312 (1992).

15. M. R. Arkin, E. D. A. Stemp, C. Turro, N. J. Turro and J. K. Barton. J. Am. Chem. Soc. 118, 2267-2274 (1996).

16. E. D. A. Stemp, M. R. Arkin and J. K. Barton. J. Ant. Chem. Soc. 117, 2375-2376 (1995).

17. M. R. Arkin, E. D. A. Stemp, R. E. Holmlin, J. K. Barton, A. Hörmann, E. J. C. Olson and P. F. Barbara. Science 273, 475-480 (1996).

18. a) E. J. C. Olson, D. Hu, A. Hörmann and P. F. Barbara. J. Phys. Chem. 101, 299-303 (1997). b) P. Lincoln, E. Tuite and B. Nordén. J. Am. Chem. Soc. 119, 1454-1455 (1997).

19. S. J. Franklin, C. R. Treadway and J. K. Barton, submitted for publication (1997).

20. S. O. Kelley, R. E. Holmlin, E. D. A. Stemp and J. K. Barton. J. Am. Chem. Soc. in press (1997).

21. D. B. Hall, R. E. Holmlin and J. K. Barton. Nature 382, 731-735 (1996).

22. a) D. T. Breslin and G. B. Schuster. J. Am. Chem. Soc. 118, 2311-2319 (1996). b) I. Saito, M. Takayama, H. Sugiyama and K. Nakatani. ibid. 117, 6406-6407 (1995). c) H. Kasai, Z. Yamaizumi, M. Berger and J. Cadet. ibid. 114, 9692-9694 (1992). d) K. Ito, S. Inoue, K. Yamamoto and S. Kawanishi. J. Biol. Chem. 268, 13221. 13227 (1993).

23. M. E. Nunez and J. K. Barton, unpublished results

24. D. B. Hall and J. K. Barton. J. Am. Chem. Soc. 119, 5045-5046 (1997).

25. a) I.-J. Chang, H. B. Gray and J. R. Winkler. J. Am. Chem. Soc. 113, 7056-7057 (1991). b) N. Liang, A. G. Mauk, G. J. Pielak, J. A. Johnson, M. Smith and B. M. Hoffman. Science 240, 311-313 (1988).

E. D. A. Stemp, M. R. Arkin and J. K. Barton. J. Am. Chem. Soc. 119, 2921-2925 (1997).

M. R. Arkin, E. D. A. Stemp, S. C. Pulver and J. K. Barton. Chemistry and Biology 4, 389-400 (1997).

A. Sancar, Biochemistry 33, 2-9 (1994).

T. Young, R. Nieman and S. D. Rose. Photochem. Photobiol. 52, $661-668$ (1990).

C. Turro, A. Evenzahav, S. H. Bossmann, J. K. Barton and N. J. Turro. Inorg. Chim. Acta 243, 101-108 (1996).

P. J. Dandliker, R. E. Holmlin and J. K. Barton. Science 274, 1465-1468 (1997). 